\title{
Analysis of Digital Rights Management for the Consumer Behaviors and the Implications for the Digital Content Industry
}

\author{
XIONG Li, CHEN Peng ${ }^{1}$
}

\begin{abstract}
With the rapid development of ICT technologies, the digital content copyright infringement has become more and more convenient and common. As a result, DRM (digital rights management) are introduced in order to protect all kinds of digital content from being pirated. This paper researches the controversial DRM and its impacts on consumer infringements. Firstly, the utility and the function of consumer behavior is analyzed and constructed as well as digital content market welfare under digital rights management environment. Secondly, the effects of different factors, such as price, valuation and pirate penalty on consumer behaviors and their relationships with market profits are studied elaborately. Finally, we draw conclusions as well as suggestions that are helpful to alleviate digital content pirate and manage the digital rights effectively.
\end{abstract}

Keywords: information consumption. digital content. digital right management.

\subsection{Introduction}

With the widespread deployment and application of the broadband and wireless networks, the global digital content market is growing exponentially [1], PricewaterhouseCoopers forecasts that the global entertainment and media market will be 1.9 trillion by the end of the year 2015 from 1.4 trillion in 2010 at an impressive average annual growth rate of 6 percent [2]. Compared with the traditional kinds of content product market, digital content product market is a new ecosystem for the core value and the carrier [3]. They have completely great differences in the production, storage, distribution and other links that compose the value chain [4], For example, digital content products can be spread via the Internet and different types of digital content products can be merged to form new product, service and market.

\footnotetext{
${ }^{1}$ XIONG Li, CHEN Peng $(\bowtie)$

School of Management, Shanghai University, 200444 Shanghai, China e-mail: chenpeng2009@shu.edu.cn
} 
The digital content market has been developing rapidly all the recent years because digital content products are easy to be copied, modified and redistributed [5], but this is a double-edged sword, which has been jeopardized the interest of digital content providers, distributors, platform operators and other stakeholders for content product copied and re-distributed without authority. In consideration of the traditional format content, the digital content infringement and pirate is more harmful especially on peer-to-peer network [6], ubiquitous computing [7], multimedia social network [8] and mobile network [9]. DRM refers to the technologies designed to control how end users can access, copy, or convert information goods [10], which aim to provide full digital content products life cycle protection [11] and ensure the legitimate use [12]. The industry believes that DRM technologies decrease pirate and without DRM protection piracy volumes would grow just because it would become easier to copy illegally.

\subsection{Controversies and Effects}

Digital rights management (DRM) has had a controversy history. It has been called "the savior of intellectual property rights" and "completely useless" in protecting digital content.

\subsubsection{Controversies over DRM}

In digital times, digital contents are being increasing consumed over the internet. That's why the role of DRM becomes more and more essential as it's perceived to provide a safe environment for transaction of copyrighted content in the networked world by preventing access to it without the content provider's authorization, making consumer's rights explicit and thereby establishing new channels for revenue generation. DRM has been a highly controversial topic since its inception in the developed countries in the 1990s. Apple Inc. former CEO, Steve Jobs's letter entitled Thoughts on Music, suggesting that record labels should sell songs online unencumbered by DRM, has caused quite a stir within the music industry and consumer groups in Europe and the USA. It has once more brought to the forefront debates surrounding DRM.

DRM is full of controversies for it involves mediation of conflicting interests, involving significant investments of various stakeholders in the industry. The main interest groups are the content creators and copyright owners, wholesalers and retailers of content, network providers, manufacturers of consumer electronics, computer hardware, DRM developers and vendors, consumers and social stakeholders [13]. For example, the consumers try to search a pirated version of the product without DRM after they find DRM are difficult to operate, 
lack interoperability, limit legitimate exceptions, invade their privacy and restrict the access and use [14]. In order to safeguard their interests the other stakeholders resort to more strict technical limitations thus a vicious circle of pirate is created [15].

\subsubsection{Effects of DRM on Consumers}

DRM not only prohibits consumers to format shift digital content legitimately, but also impinges upon their freedom of expression and inhibits their non-conformist opinions by forbidding content use for academic research, reporting in media, review, criticism, etc., that are permissible under the Fair use or Fair dealing doctrines [16].

Concerns pertinent from the privacy perspective include the extent to which DRM will collect and further process the consumers' personal data while browsing or buying digital content online, the purposes for and the conditions under which such data will be used and sold to others. The privacy of consumers may be affected if DRM assists in the processing of these data about them without their consent or knowledge, etc. Consumers believe that their integrity and privacy could be offended if such processing does not conform to their expectations of what is reasonable or not. The tension between DRM and privacy issues is heightened in cases of reuse of such data for secondary purposes [17]. As a result, there are major differences between digital contents with DRM and digital contents without DRM in valuation for consumers.

The existing literature has demonstrated (e.g., Pfeiffer Consulting 2001, IDC 2001, Berry 2002 and Demon 2006 [18]) that consumer prefers the unrestricted product version to the restricted one, which is protected by DRM. The consumer's valuation of digital content is $\theta_{p}$ when it's DRM-free and the valuation is $\theta_{b}$ for the DRM-protected. We can derive the following inequation:

$$
\theta_{p}>\theta_{b}
$$

Compared to an unrestricted version, the disutility of inconvenience consumers must experience when having the DRM protected products is

$$
\Delta \theta=\theta_{p}-\theta_{b}
$$

\subsection{The Model}

This section details the main model of the paper used to analyze the impact of DRM restrictions on the level of piracy and on the prices and profits of different 
members in the digital content industry. We start with the products and consumers, and then continue with the market which is another important part that consists of the digital content industry chain.

\subsubsection{Products}

We assume that there are two kinds of digital content on the market, the DRMprotected and the DRM-free. The former is provided by the legitimate providers only and can be obtained legally by buying while the later is illegally from a variety of sources such as p2p network, pirated website, a friend, etc. The market is completely covered by these two kinds of digital content such that each consumer either buys or pirates at most one unit of the product because there is no additional value from obtaining a second unit in digital format.

\subsubsection{Consumers}

Different consumers have different preferences for digital content in DRMprotected versus DRM-free. There can be various reasons underlying such preference: some consumers may like high quality and legitimacy of the DRMprotected digital content, while others may prefer the DRM-free ones for unrestricted access and use, or the low cost spent for copying or searching for an illegal copy.

Ultimately, in order to maximize his utility, each consumer makes a choice of which kind to obtain and the choice depends on consumer's preference and price of each product. In other words, consumers consider these two kinds of digital content as horizontally differentiated products.

The providers sell the digital content to the consumers at price $p_{\text {dc }}$ for each unit. The expected fine that consumers would pay if caught stealing is $c_{p l}$ and the probability is $\rho$ distributed on segment $[0,1]$. The percentage of consumers that pirate is $\lambda$ which is also distributed on segment $[0,1]$. The utility of each consumer can be expressed as the following

$$
U_{C}^{i}=\left\{\begin{array}{r}
\theta_{b}-p_{d c}, i \in B \\
\theta_{p}-\lambda \cdot \rho \cdot c_{p l}, i \in P
\end{array}\right.
$$

The symbols B, P represent buying the digital content and pirating the digital content respectively.

When any one of the consumers compares the utility of buying a restricted, DRM-protected product with the utility of pirating an unrestricted, DRM-free 
digital product, the result of the comparison does not depend on the consumer's preferences and other factors only if

$$
U_{C}^{B}-U_{C}^{P}=0
$$

So the equilibrium proportion for DRM-free product is

$$
\lambda=\frac{\Delta \theta+p_{\mathrm{dc}}}{\rho \cdot c_{p l}}
$$

Therefore, the larger $\Delta \theta$ and $p_{d c}$, the more likely it is that the equilibrium proportion of the consumers that choose to pirate ascends. But contrarily, it is just the opposite when $\rho$ and $c_{p l}$ become larger.

\subsubsection{Market}

Consider a setting that consists of one digital content provider, and consumers who may buy or pirate digital products. The digital content provider sells the products to the consumers at a specific price in order to earn profit.

\subsubsection{Provider's Benefit}

The market is completely covered by DRM-protected digital contents and DRMfree digital contents. There are $1-\lambda$ consumers prefer buying the DRM-protected version when there are $\lambda$ consumers prefer to steal the DRM-free digital product. Together with (1.8), the provider's profit is

$$
W_{s}=p_{d c}\left(1-\frac{\Delta \theta+p_{d c}}{\rho \cdot c_{p l}}\right)
$$

The necessary condition for profit maximization is

$$
\frac{\partial W_{S}}{\partial p_{d c}}=1-\frac{\Delta \theta+p_{d c}}{\rho \cdot C_{p l}}-\frac{p_{d c}}{\rho \cdot C_{p l}}=0
$$

The unique optimal price that maximizes the provider's benefit is given by

$$
p_{d c}^{*}=\frac{\rho \cdot c_{p l}-\Delta \theta}{2}
$$

We formulate the profit maximization problem for the providers as following:

$$
W_{S}^{*}=\frac{1}{\rho \cdot c_{p l}}\left(\frac{\rho \cdot c_{p l}-\Delta \theta}{2}\right)^{2}
$$




\subsubsection{Consumer's Utility}

The consumers can obtain utility whether buying from the legal providers or pirating from other distributers that are not authorized. But it leads to a deadweight loss of market welfare on the behalf of the providers and other stakeholders when consumer decides to pirate not to buy.

$$
W_{i}=\left\{\begin{array}{rr}
(1-\lambda) U_{C}^{i}, & i \in B \\
-\lambda U_{C}^{i}, & i \in P
\end{array}\right.
$$

\subsubsection{Market Welfare}

The digital content market welfare $W$ derived by the providers and the consumers is given by

$$
W=W_{S}+W_{B}+W_{P}=\frac{\left(\rho \cdot c_{p l}-\Delta \theta\right)^{2}}{4 \rho \cdot c_{p l}}+\frac{\left(\theta_{b}+\theta_{p}\right)}{2 \rho \cdot c_{p l}} \cdot \Delta \theta
$$

In the following, we provide numerical results to illustrate the impacts of the price, valuation and penalty on the consumer behaviors. The results are showed in the below figure.

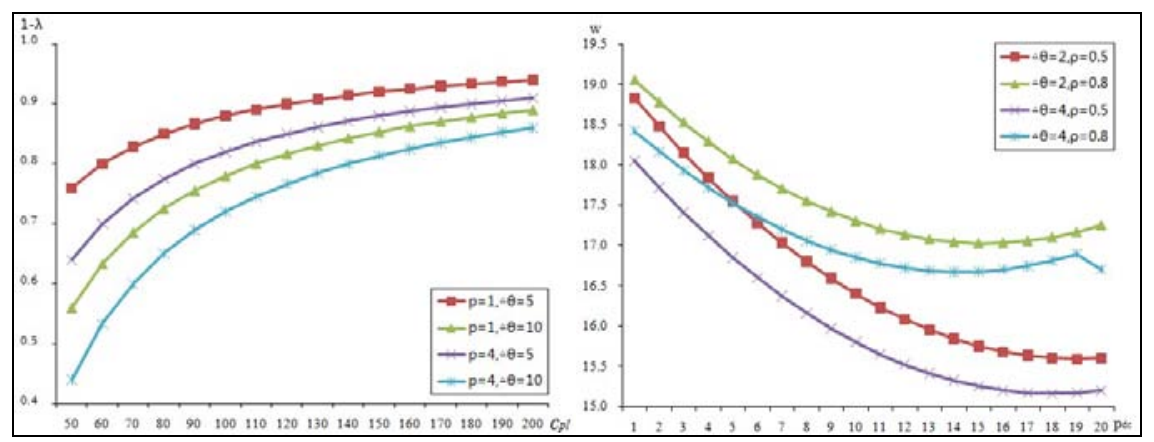

Fig. 1.1 the numerical results of price, valuation and pirate penalty on the consumer behaviors and the market welfare

\subsection{Conclusions}

In this thesis, we investigate the digital content consumer behaviors under DRM and explore the major effects of price, valuation and pirate penalty on consumer 
behaviors as well as their relationships with market welfare. Finally, we arrive at the following conclusions:

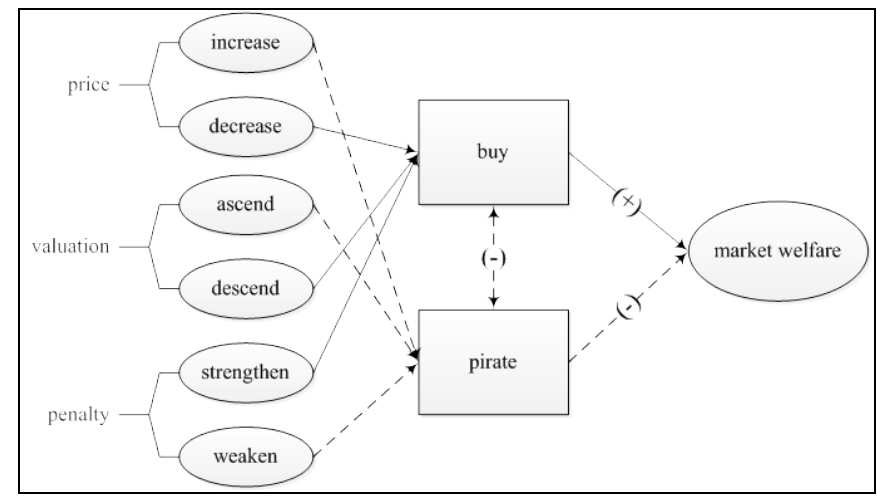

Fig. 1.2 the effects of different factors, such as price, valuation and pirate penalty on consumer behaviors and their relationships with market welfare

- All of the three factors mentioned above have significant impacts on consumer behavior, especially the tort penalty probability. The market equilibrium adjusts itself to any one of the factors while the others keep fixed. To be specific, less and less consumer would pirate as long as digital content product price becomes lower even though the other factors remain unchanged.

- Buying and pirating reflect the law of unity of opposites in nature and human society and there is always a dynamic balance between them. Not only the digital content market welfare but also the whole industry is heavily influenced by the consumer behaviors. Specifically, the more digital content products consumers choose to buy in, the greater welfare it is to some extent.

- DRM tends to intensify pirating for reducing the consumers' valuation of the digital content, particularly when there is no perfect or robust legal system. It means that the consumer infringement is not a problem that could be solved only by techniques such as DRM. The consumer behaviors should be constrained and guided with the assistance of laws which is helpful to alleviate pirating and manage the digital rights effectively.

\subsection{Acknowledgment}

This research is supported by the Research Planning Funds Project on Humanities and Social Science of Ministry of Education (No. 12YJA630158) and Shanghai Philosophy and Social Sciences Planning Funds Project (NO. 2011BTQ001). The authors are very grateful to the editors and anonymous reviewers for the thorough comments that greatly improved and enhanced the quality of this paper. 


\subsection{References}

1. Kim Changsu, Oh Eunhae, Shin Namchul (2010) An empirical investigation of digital content characteristics, value, and flow. Journal of Computer Information Systems, 50(4): 79-87

2. PricewaterhouseCoopers (2011) Global entertainment and media outlook (2011-2015) http://w ww.pwc.com/us/em/outlook

3. Bockstedt Jesse, Kauffman Robert, Riggins Frederick (2006) The move to artist-led on-line music distribution: a theory-based assessment and prospects for structural changes in the digital music market. International Journal of Electronic Commerce, 10(3): 7-38

4. Liu Zhuo-jun, Zhou Cheng-xiong (2006) The effect of Internet environment on market of digital content product. Chinese Journal of Management Science, 14(4): 95-99

5. Jin Bih-Huang, Li Yung-Ming (2012) Analysis of emerging technology adoption for the digital content market. Information Technology and Management 13(3): 149-165

6. Tsolis Dimitrios, Sioutas Spyros, Xenos Michalis, Styliaras Georgios (2011) Copyright and IPR management for cultural heritage digital content in peer-to-peer networks. Journal of Cultural Heritage 12(4): 466-475

7. Akihiro Tsutsui, Tomoko Sawabe (2011) Secure method of managing digital content in ubiquitous computing environment. NTT Technical Review, 9(10): 1-6

8. Zhang Zhiyong (2012) Frontier and methodologies on Digital Rights Management for Multimedia Social Networks. International Journal of Digital Content Technology \& Its Applications, 6(9): 245-249

9. Wu Chia-Chi, Lin Chia-Chen, Chang Chin-Chen (2010) Digital rights management for multimedia content over 3G mobile networks. Expert Systems with Applications, 37(10): 6787-6797

10. Xue Mei, Yuan Zhong-xiong (2011) Research on digital content whole lifecycle protection model. Computer Engineering, 37(23): 33-37

11. Yu Yin-yan, Tang Zhi (2005) A survey of the research on Digital Rights Management. Chinese Journal of Computers, 28(12): 1957-1968

12. Fan Ke-feng, Mo Wei, Cao Shan, Zhao Xin-hua, Pei Qing-qi (2007) Advances in Digital Rights Management technology and application. Acta Electronica Sinica, 35(6): 1139-1147

13. Joan Tassel (2006) Digital Rights Management: Protecting and Monetizing Content. Focal Press

14. Ma Guo-jun, Pei Qing-qi, Jiang Xiao-hong (2012) Analysis of DRM game control. Journal on Communications, 33(Z1): 282-286

15. Habtamu Abie (2007) Frontiers of DRM Knowledge and Technology. International Journal of Computer Science and Network Security, 7(1): 216-231

16. Sensarkar Nilanjana (2007) The potential impact of digital rights management on the Indian entertainment industry. Journal of International Trade Law and Policy, 6(1): 45-55

17. Ma Zhao-feng, Fan Ke-feng, Chen Ming, Yang Yi-xian, Niu Xin-xin (2008) Trusted digital rights management protocol supporting for time and space constraint Journal on Communications, 29(10): 153-164

18. Domon Koji (2006) Price discrimination of digital content. Economics Letters, 97(1): 421426 